\title{
Change in Impedance with Change in Posture during Spinal Cord Stimulator Placement
}

\author{
Samer Abdel-Aziz, Ahmed Ghaleb \\ Department of Anesthesiology and Pain Medicine, University of Arkansas for Medical Sciences, Little Rock, USA \\ Email: sabdelaziz@uams.edu
}

Received 18 January 2014; revised 20 February 2014; accepted 25 March 2014

Copyright (C) 2014 by authors and Scientific Research Publishing Inc.

This work is licensed under the Creative Commons Attribution International License (CC BY). http://creativecommons.org/licenses/by/4.0/

c) (i) Open Access

\begin{abstract}
In this article we discuss the effect of posture on impedance during the placement of a spinal cord stimulator. Although, according to several studies, there appears not to be a specific and consistent change in impedance with change in posture, we noticed on multiple occasions that if the impedance remains very high during spinal cord stimulator (SCS) lead placement, asking the patient to sit up from the prone position may drop the impedance down. This could be explained by having air around the lead, from using loss of resistance to air technique in finding the epidural space, which moves up when sitting the patient up, or having the lead immersed in epidural fat. Sitting the patient up from the prone position should be tried if the impedance remains very high before aborting the procedure and taking the lead out.
\end{abstract}

\section{Keywords}

Impedance, Spinal Cord Stimulator, Change in Posture

\section{Introduction}

We have noticed in many cases while placing spinal cord stimulators (SCS) that the impedance would be very high when the patient was in the prone position and dropped down when the patient was asked to sit up, here we report one example.

\section{Case Description}

A 73-year-old female patient with lower back pain for several years, her pain radiated to her right lower extremity, was on average 8/10 in severity and was affecting the quality of her life. She tried multiple medication regi- 
mens and had multiple epidural steroidal injections with minimal relief. Decision was made to proceed with a SCS trial. A 14 gauge Tuohy needle was introduced using a right paramedian approach, advanced into the epidural space at L1-L2 level at an angle of 30 - 45 degrees to the skin. The epidural space was confirmed using a loss of resistance to air technique and by passing a guide wire. The SCS lead was advanced with maneuvering, positioned slightly right to the center over the dorsal column of the spinal cord. The most cephalic electrode was placed at the level of the T7 vertebral body. A second SCS lead was introduced and advanced using the same technique on the left side, the most cephalic electrode was also placed at the level of the T7 vertebral body (Figure 1). The leads were then connected to the programming extension and passed to the company representative. Upon testing, while the patient was in the prone position, we noticed that the impedance read from both leads was very high $(>10,000 \mathrm{ohm})$. We tried to move the leads and changed the programming extension however the impedance remained (>10,000 ohm), after everything we tried failed to get the impedance down we sutured the leads to the skin and asked the patient to sit up, once the patient sat up the impedance dropped to around $3500 \mathrm{ohm}$. The SCS was programmed to give the patient a good pain coverage and was fixed to the patient.

\section{Discussion}

This case describes a very significant change in the impedance read from the SCS leads when the patient changed her position from prone to sitting during SCS placement. The change was very significant, from $>10,000$ ohm to $3500 \mathrm{ohm}$, which would make the difference between a non functional SCS and a functional SCS.

Several studies have reported a change in impedance with the change in the patient's position. Cameron et al.

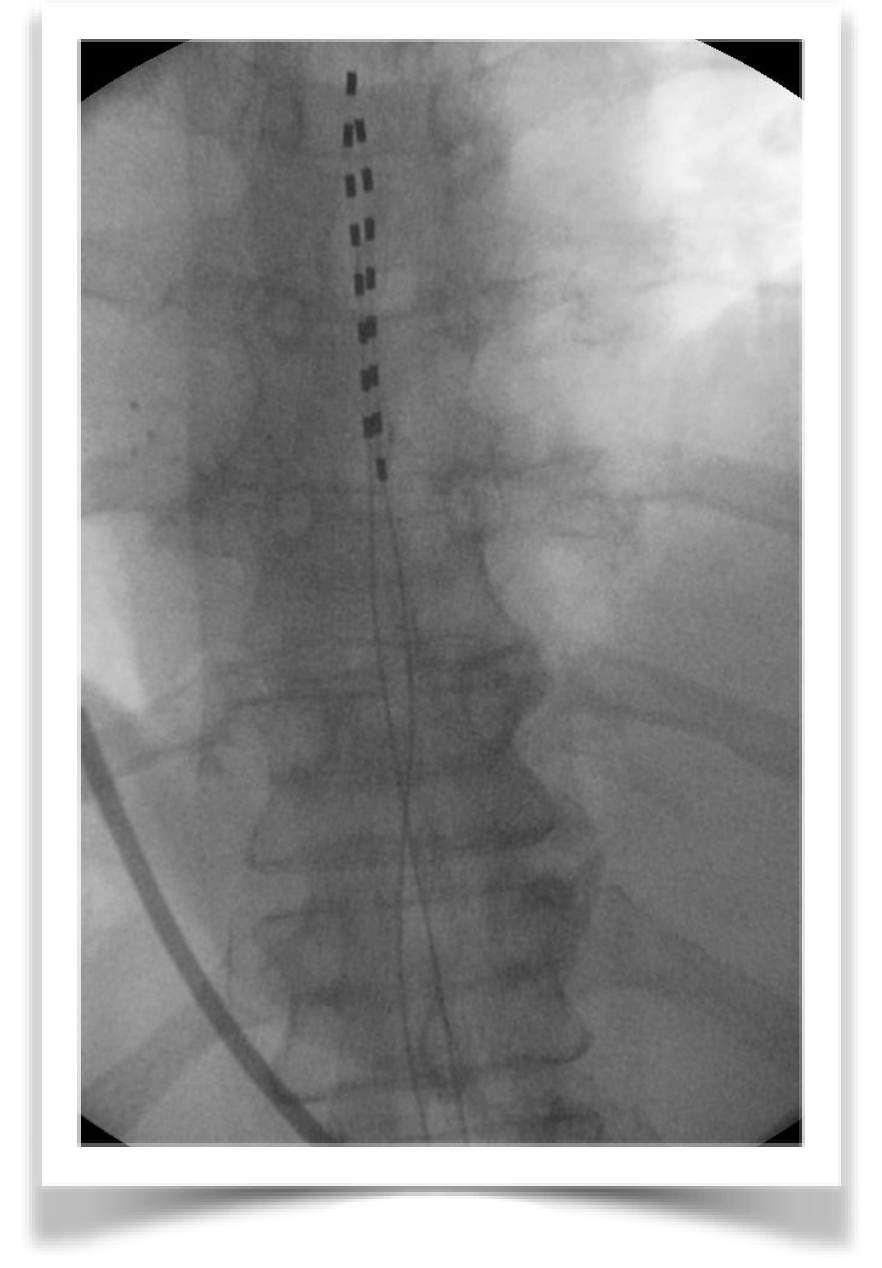

Figure 1. Two low thoracic spinal cord stimulator leads. 
examined the importance of posture on the efficacy of spinal cord stimulation in 29 chronic pain patients previously implanted with a SCS, they found that in 20 patients the threshold for paresthesia was lowest when lying, while In three patients it was lowest when sitting. They also found that stimulation required to achieve paresthesia was found to be lower for leads in the cervical region when compared with leads in the thoracic region [1]. Olin et al. obtained voltage measurements at the threshold of perception in three body postures for patients with percutaneous dorsal epidural leads in 42 patients. They observed a significant increase in voltage requirements when moving from supine to sitting or standing positions [2]. Alo et al. characterized the typical impedance values measured from common quadripolar percutaneous SCS contact arrays in 36 patients undergoing percutaneous trial stimulation for various chronic pain conditions. They found that the median impedance in the cervical region was significantly lower than in the lower-thoracic region [3]. Abejon et al. studied the relationship between changes in impedance and energy requirement elicited by changes in patient's posture. In 70 patients with chronic pain, they analyzed changes in impedance and energy requirement in supine, sitting, standing and walking postures. They found that the supine position was the posture requiring the lowest energy and that electrodes implanted in the cervical region required less energy, however these differences were not statistically significant [4].

There seems to be an agreement that change in posture can in some cases cause a change in impedance in patients with SCS, although this change can be insignificant or inconsistent. There is also an agreement that impedance in the thoracic area tends to be higher than in the cervical area, this could be due to the dorso-ventral position of the lead in the epidural space. The presence of a larger space between the triangularly shaped dorsal part of the vertebral arch and the round shape of the dural sac in the lower-thoracic region increases the likelihood that the stimulating lead will not make dural contact, and thus "see" an increased impedance from the surrounding epidural fat [3].

The main factors that cause an increase in impedance while placing the SCS lead are; epidural fat around the lead, air around the lead and lead disconnection or malfunction. If epidural fat surrounding the lead is suspected, maneuvering the lead might free it from the surrounding fat, however while controlling the cephalo-caudal and medio-lateral position of the lead is possible, the dorso-ventral position is very difficult to control, sitting the patient up from the prone position may cause the lead to move in the dorso-ventral space and result in a change in the impedance. Another main factor is air around the lead, if loss of resistance to air technique was used to find the epidural space and specially if a lot of air was injected, air could get trapped around the lead isolating it from the dura and causing high impedance reads, sitting the patient up from the prone position moves the air up thus decreasing the impedance. Lead disconnection or malfunction is another possibility, however before taking the lead out, we recommend sitting the patient up from the prone position, it is a simple thing worth of trying before aborting or repeating the procedure.

\section{Conclusion}

Air and epidural fat around the lead are factors that cause high impedance while placing the SCS, sitting the patient up from the prone position may free the lead from surrounding air (air moves up), or surrounding epidural fat (lead moves in the dorso-ventral space). It is worth trying before assuming a faulty lead and repeating the procedure.

\section{References}

[1] Cameron, T. and Alo, K. (1998) Effects of Posture on Stimulation Parameters in Spinal Cord Stimulation. Neuromodulation, 1, 177-183. http://dx.doi.org/10.1111/j.1525-1403.1998.tb00014.x

[2] Olin, J., Kidd, D. and North, R. (1998) Postural Changes in Spinal Cord Stimulation Perceptual Thresholds. Neuromodulation, 1, 171-175. http://dx.doi.org/10.1111/j.1525-1403.1998.tb00013.x

[3] Alo, K., Varga, C., Krames, E., Prager, J., Holsheimer, J., Dipl, L.M. and Bradley, K. (2006) Factors Affecting Impedance of Percutaneous Leads in Spinal Cord Stimulation. Neuromodulation, 9, 128-135. http://dx.doi.org/10.1111/j.1525-1403.2006.00050.x

[4] Abejon, D. and Feler, C.A. (2007) Is Impedance a Parameter to Be Taken into Account in Spinal Cord Stimulation. Pain Physician, 10, 533-540. 